\title{
Critical role for a high-affinity chemokine-binding protein in $\gamma$-herpesvirus-induced lethal meningitis
}

\author{
Victor van Berkel, ${ }^{1}$ Beth Levine, ${ }^{2}$ Sharookh B. Kapadia, ${ }^{1}$ James E. Goldman, ${ }^{3}$ \\ Samuel H. Speck, ${ }^{4}$ and Herbert W. Virgin IV ${ }^{1}$ \\ ${ }^{1}$ Departments of Pathology and Immunology and Molecular Microbiology, Washington University School of Medicine, \\ St. Louis, Missouri, USA \\ ${ }^{2}$ Department of Medicine, and \\ ${ }^{3}$ Department of Pathology, Columbia University College of Physicians and Surgeons, New York, New York, USA \\ ${ }^{4}$ Department of Microbiology and Immunology, Yerkes Regional Primate Center, Emory University, Atlanta, Georgia, USA \\ Address correspondence to: Herbert W. Virgin IV, Department of Pathology, \\ Box 8118, 660 South Euclid Avenue, St. Louis, Missouri 63110, USA. \\ Phone: (314) 362-9223; Fax: (314) 362-4096; E-mail: virgin@immunology.wustl.edu.
}

Received for publication October 5, 2001, and accepted in revised form March 5, 2002.

\begin{abstract}
Chemokines are involved in recruitment and activation of hematopoietic cells in sites of infection and inflammation. The $M 3$ gene of the $\gamma$-herpesvirus $\gamma \mathrm{HV} 68$ encodes an abundant secreted protein that binds $\mathrm{CC}$ chemokines with high affinity. We report here that this gene is essential for efficient induction of lethal meningitis by $\gamma \mathrm{HV} 68$. An M3 mutant $\gamma \mathrm{HV} 68$ ( $\gamma \mathrm{HV} 68$-M3.stop) was 100 -fold less virulent than wild-type or marker rescue control $(\gamma \mathrm{HV} 68-\mathrm{M} 3 . \mathrm{MR})$ viruses after intracerebral inoculation. After intracerebral inoculation, $\gamma \mathrm{HV} 68-\mathrm{M} 3$.stop grew to lower titers than $\gamma \mathrm{HV} 68$ or $\gamma \mathrm{HV} 68$-M3.MR in the brain but spread to and grew normally in the spleen and lung. Expression of several CC chemokines was significantly induced in the CNS by $\gamma \mathrm{HV} 68$ infection. Consistent with M3 acting by blockade of $\mathrm{CC}$ chemokine action, $\gamma \mathrm{HV} 68$ induced a neutrophilic meningeal inflammatory infiltrate, while $\gamma$ HV68-M3.stop induced an infiltrate in which lymphocytes and macrophages predominated. In contrast to the important role of M3 in lethal meningitis, M3 was not required for establishment or reactivation from latent infection or induction of chronic arteritis. These data suggest a role for chemokines in the protection of the nervous system from viral infection and that the M3 protein acts in a tissue-specific fashion during acute but not chronic $\gamma \mathrm{HV} 68$ infection to limit CC chemokine-induced inflammatory responses.
\end{abstract}

J. Clin. Invest. 109:905-914 (2002). DOI:10.1172/JCI200214358.

\section{Introduction}

Chemokines are chemoattractant and immunomodulatory molecules that play a central role in many inflammatory processes (1). Chemokines are divided into four structural groups based on the number and arrangement of conserved cysteines and are consequently named CC, $\mathrm{CXC}, \mathrm{C}$, and $\mathrm{CX}_{3} \mathrm{C}$ chemokines. $\mathrm{CC}$ chemokines generally regulate macrophages and lymphocytes, while CXC chemokines stimulate the activity of neutrophils as well as regulate lymphocyte activity and development. The only members of the $\mathrm{C}$ and $\mathrm{CX}_{3} \mathrm{C}$ chemokine families are lymphotactin and fractalkine, respectively, and their physiologic roles are not completely understood.

Given the importance of chemokines in the immune system, it is not surprising that viruses have evolved mechanisms for interacting with the chemokine system. Poxviruses and herpesviruses use multiple strategies for interacting with the chemokine system, including virus-encoded chemokine receptor homologues and virus-encoded chemokine homologues (2-6). An additional strategy, secretion of chemokine-binding proteins, was originally described in poxviruses $(5,6)$ and has subsequently been demonstrated for the $\gamma$-herpesvirus, $\gamma \mathrm{HV} 68(7,8)$.
Two distinct types of DNA viruses, poxviruses and herpesviruses, express high-affinity chemokine-binding proteins (CBPs). Poxvirus CBPs are involved in regulating inflammation during acute infection. Myxoma viruses deficient in production of the CBP T7 have dramatic reduction in disease symptoms and viral dissemination to secondary sites, as well as a marked increase of leukocyte infiltration into the site of infection (9). Myxoma viruses deficient in CBP T1 have a more subtle phenotype, with an increase in leukocyte infiltration, but no significant difference in disease progression or mortality $(10,11)$.

Limitation of inflammatory chemokine action during acute infection may be similarly important for the pathogenesis of disease caused by herpesviruses. However, $\gamma$-herpesviruses have a unique relationship with hematopoietic cells that is not shared with poxviruses. Unlike poxviruses, $\gamma$-herpesviruses require hematopoietic cells for latency and long-term persistence in the host and are capable of causing chronic diseases including lymphomas and arteritis of the great vessels (12, 13). It is therefore possible that chemokines and CBPs play a role in acute $\gamma$-herpesvirus infection (similar to poxviruses) and or chronic $\gamma$-herpesvirus infection. 
$\gamma \mathrm{HV} 68$ is a $\gamma 2$-herpesvirus with homology to EpsteinBarr virus (EBV), herpesvirus saimiri (HVS), and Kaposi sarcoma herpesvirus (KSHV, HHV8). $\gamma \mathrm{HV} 68$ infects laboratory mice and can be genetically manipulated $(14,15)$, providing a unique tool for identifying host and viral factors that regulate $\gamma$-herpesvirus pathogenesis (reviewed in refs. 12, 13). The $\gamma H$ HV6 M3 gene encodes an abundant secreted protein (16) that is capable of binding to a broad spectrum of chemokines and blocks chemokine signaling $(7,8)$. To date, M3 binds murine CC chemokines with about 100-fold higher affinities than murine CXC chemokines (8), suggesting that M3 may selectively block certain classes of chemokines in vivo. To examine the biologic role of this chemokine-binding protein in herpesvirus infection, we constructed a recombinant $\gamma$ HV68-M3.stop mutant virus that is deficient in production of M3 protein. We report here that the $\mathrm{M} 3$ protein plays a critical role in acute viral meningitis, but does not have a role in chronic vasculitis or in the establishment of, or reactivation from, latency.

\section{Methods}

Viruses and tissue culture. $\gamma$ HV68 WUMS (ATCC VR1465) was used for all infections and is designated as wild-type $\gamma \mathrm{HV} 68$. $\gamma \mathrm{HV} 68$ was passaged on NIH $3 \mathrm{~T} 12$ cells for amplification and was propagated as described previously (17). NIH 3T12 cells and mouse embryonic fibroblasts (MEFs) were maintained in DMEM, supplemented with $10 \%$ FCS, $100 \mathrm{U} / \mathrm{ml}$ penicillin, $100 \mathrm{U} / \mathrm{ml}$ streptomycin, and $2 \mathrm{mM}$ L-glutamine (complete DMEM). Cells were maintained in a $5 \% \mathrm{CO}_{2}$ tissue culture incubator at $37^{\circ} \mathrm{C}$. MEFs were obtained from either BALB/c or C57BL/ 6 mouse embryos as described previously (17).

Generation of virus mutants. The $\gamma \mathrm{HV} 68-\mathrm{M} 3$.stop virus was generated by Lipofectamine cotransfection of $\gamma$ HV68 genomic DNA (18) with a gene-targeting plasmid. The parental targeting vector, M3-64, contains the fragment of the $\gamma \mathrm{HV} 68$ genome from the SpeI site at genomic coordinate 4632 to the StuI site at 8242 inserted into the cloning vector Litmus 28. The $\gamma \mathrm{HV} 68-$ M3.stop targeting vector was generated by inserting a linker sequence into the AccI site at 7176. The stop linker was formed by annealing the following oligos: $5^{\prime}$ CTACCTAGGACTAAGT- $3^{\prime}$ and $5^{\prime}$-AGACTTAGTCCTAGGT$3^{\prime}$. This linker inserts three in-frame translational stop codons, a frameshift mutation, and a novel AvrII site.

Virus was harvested 6 days after transfection, diluted, plated on NIH 3T12 monolayers, and after an hour of infection, overlaid with methylcellulose $(2 \%$ methylcellulose in DMEM supplemented with 5\% FCS, antibiotics, and L-glutamine) as described previously (15). One hundred plaques were picked from each transfection and resuspended in $0.5 \mathrm{ml}$ of complete DMEM. Five microliters of the resuspended plaque were subjected to proteinase $\mathrm{K}$ digestion overnight and then analyzed by nested PCR specific for the engineered mutation (outside primers: 5'-AGAGGGGAATGGCAATCTGCCTGTT-3', 5'-GCATATTTAAGGAGGGTCTCCCT-
GC-3'; inside primers: 5'-CCTTCCTATCCACATCTGTGCTCAT-3' 5' $^{\prime}$-AAGAGTGG-GTAGACTTAGTCCTAGG-3'). Positive recombinants were detected with a frequency of approximately $4 \%$ in the first round of plaque purification. Plaques containing mutant virus were plaque purified until homogenous (three rounds).

Small viral stocks were prepared for DNA analysis as described previously (15). To assess for the presence of the engineered mutation, Southern analysis was performed with a diagnostic AvrII digest and a ${ }^{32} \mathrm{P}$-labeled M3 region probe (bp 5362-7893 of $\gamma \mathrm{HV} 68$ WUMS). Large viral stocks were generated as described previously (15), and titers were independently determined by plaque assay three times. Western blot analysis was performed as described previously (16).

The M3 marker rescue virus was generated by cotransfection of the parental M3-containing plasmid (M3-64) with viral genome derived from the $\gamma H$ H68-M3.stop viral stock and plaque purification as above, with the exception that recombinant plaques were now identified by assessing for the presence of secreted M3 protein by ELISA. One hundred microliters of resuspended plaque were added to NIH 3 T12 monolayers in 96-well plates and incubated at $37^{\circ} \mathrm{C}$ for 6 days, at which point there was significant viral cytopathic effect. Supernatants from these infected monolayers were used to coat an Immulon II ELISA plate (Thermo Labsystems, Franklin, Massachusetts, USA) overnight at $4^{\circ} \mathrm{C}$. The ELISA was performed with rabbit antisera raised against bacterially expressed M3 protein (8) and developed with a horseradish peroxidase-conjugated secondary $\mathrm{Ab}$ specific to rabbit immunoglobin. The ELISA was capable of detecting M3 protein in a $1: 10^{6}$ dilution of supernatants infected with a wild-type resuspended plaque. Recombinant viruses were detected with a frequency of approximately $4 \%$ in the first round of purification. Generation of the $\gamma \mathrm{HV} 68-\mathrm{M} 2$.stop virus is described elsewhere (19).

Multistep in vitro growth analysis of mutant and wild-type $\gamma H V 68$ viruses. NIH 3T12 cells were infected with either $\gamma$ HV68-M3.stop or wild-type $\gamma \mathrm{HV} 68$ viruses at a moi of 0.05 in six-well plates to measure multiple cycles of virus replication. Total infected cell lysates were harvested at 12, 24, 48, 72, and 96 hours after infection, as described previously $(15,20)$.

Real-time RT-PCR analysis of mutant and wild-type $\gamma H V 68$ viruses. The effect of the M3 mutation on transcription of the adjacent M2 and M4 genes was determined using real-time PCR analysis. Transcripts from gene 6 , encoding the single-stranded DNA-binding protein, were quantitated as an internal control for viral RNA recovery. NIH 3T12 cells were infected with either wild-type $\gamma \mathrm{HV} 68, \gamma \mathrm{HV} 68-\mathrm{M} 3$. stop, or $\gamma \mathrm{HV} 68-\mathrm{M} 3 . \mathrm{MR}$ at moi $=5$, and total RNA was harvested 18 hours after infection (TRIzol, Life Technologies Inc., Grand Island, New York, USA). Two micrograms of total RNA were incubated with $200 \mathrm{ng} / \mu \mathrm{l}$ oligo-dT and $50 \mathrm{ng} / \mu \mathrm{l}$ of random hexamer at $68^{\circ} \mathrm{C}$ for 10 minutes, and $20.5 \mu \mathrm{l}$ of reaction mix were added containing $1 \mathrm{mM}$ dNTP, $1 \mathrm{X}$ First Strand buffer (Life Technologies Inc.), 20 mM DTT 
(Life Technologies), $2 \mu \mathrm{l}$ of RNAsin (Promega Corp., Madison, Wisconsin, USA), and 100 U Superscript II (RNAse $\mathrm{H}^{-}$) reverse transcriptase (Life Technologies Inc.). Reverse transcription was performed at $42^{\circ} \mathrm{C}$ for 1 hour, $500 \mu \mathrm{l}$ of water were added, and samples were boiled for 5 minutes and stored at $-20^{\circ} \mathrm{C}$. Real-time PCR (iCycler; Bio-Rad, Hercules, California, USA) was carried out in $25-\mu \mathrm{l}$ reactions containing $2.5 \mu \mathrm{l}$ RT product, 1X Gold buffer (Perkin-Elmer Applied Biosystems, Foster City, , USA), 1:20 volume of dimethyl sulphoxide, $0.2 \mathrm{mM}$ deoxynucleoside triphosphates, $2.5 \mathrm{mM} \mathrm{MgCl}_{2}$, 0.2 U AmpliTaq Gold (Perkin-Elmer Applied Biosystems), $160 \mathrm{nM}$ forward and reverse primers, and 1:30,000 final concentration of SYBR Green (Molecular Probes Inc., Eugene, Oregon, USA). Dilutions of plasmids containing $10^{9}-10^{1}$ copies of genes M2, M4, and gene 6 were run in parallel for use as a standard curve. PCR primers used for real-time PCR analysis are as follows: M2 (sense) $5^{\prime}$-GCCCACCTACAAGACCTTT-3' and (antisense) $5^{\prime}$-CCCACTCCACAAAACCTT-3'; M4 (sense) $5^{\prime}$ GGAGAAGATGATGATATTCCTGT- $3^{\prime}$ and (antisense) $5^{\prime}$ CAGAATAATTTCCTGGATGGA-3'; gene 6 (sense) $5^{\prime}$ ATGTCTCCCCATATTCTTGC- $3^{\prime}$ and (antisense) $5^{\prime}$ ACATGGAAGTGTTGGCTGT-3'.

Mice and inoculations. All mice were sex and age matched and infected between 7 and 9 weeks of age unless otherwise stated. C57Bl/6J mice were purchased from The Jackson Laboratory (Bar Harbor, Maine, USA). CD-1 outbred mice with litters for intracerebral inoculations were obtained from Charles River Laboratories (Wilmington, Massachusetts, USA). Mice were bred and maintained at Washington University School of Medicine in accordance with all federal and university policies. Mice infected by the intraperitoneal route of inoculation were infected with $10^{6}$ plaque-forming units (PFU) in $0.5 \mathrm{ml}$ of supplemented DMEM medium unless otherwise noted. For intranasal inoculation, mice were infected with $4 \times 10^{5} \mathrm{PFU}$ in $40 \mu \mathrm{l}$ of supplemented DMEM. For the intracerebral route of inoculation, 21-day-old CD1 or C57B1/6J mice were infected with varying doses of virus in a $10 \mu \mathrm{l}$ volume using a 50- $\mu$ l Hamilton syringe (Hamilton Co., Reno, Nevada, USA) and a 30-gauge Precision-Glide needle (Becton-Dickinson, Franklin Lakes, New Jersey, USA).

Analysis of infected mice. Plaque assays were performed as previously described $(15,20)$. Briefly, mice were sacrificed and organs were harvested into sterile, screwtop 2-ml tubes (Sarstedt Inc., Newton, North Carolina, USA) containing $1 \mathrm{ml}$ of DMEM medium and 100 $\mu \mathrm{l}$ 1-mm zirconia/silica beads (BioSpec Products Inc., Bartlesville, Oklahoma, USA) and stored at $-80^{\circ} \mathrm{C}$ until the plaque assay was performed. Samples were then thawed on ice and homogenized using the Mini BeadBeater (BioSpec Products Inc.) for 1 minute at maximum speed. One hundred microliters of homogenized organ were then used for dilutions. The limit of detection for the plaque assays was 50 PFU. Organs for pathology were collected into $10 \%$ buffered formalin, fixed for at least 72 hours, and embedded in paraffin for sectioning and staining with hematoxylin and eosin or left unstained for immunohistochemistry. Differential counts were performed in a blinded manner by two investigators.

Immunohistochemistry. Immunohistochemical staining with immunofluorescence was performed as described previously (21). Anti- $\gamma$ HV68 polyclonal rabbit serum and preimmune rabbit serum were diluted 1:1,000 and added to slides following deparaffinization and antigen retrieval, incubated overnight at $4^{\circ} \mathrm{C}$, and washed. Cy3-conjugated donkey anti-rabbit secondary $\mathrm{Ab}$ at a 1:250 dilution was added, incubated at room temperature for 1 hour, and washed. Slides were counterstained in a 1:2,000 dilution of Hoechst dye for 15 minutes, washed, covered with PBS-glycerol (1:1) coverslips, and stored at $4^{\circ} \mathrm{C}$ in the dark. No staining was observed in infected tissues incubated with preimmune serum. Similarly, no staining was observed in uninfected tissues incubated with immune serum.

$R$ Nase protection assay. RNA was harvested from half brains of individual mice using the RNeasy Midi Kit from QIAGEN Inc. (Valencia, California, USA). Five days after intracerebral injection of either DMEM as a control, 100 PFU of $\gamma$ HV68-M3.MR, or 100 PFU of $\gamma$ HV68-M3.stop, mice were sacrificed, and half of the brain was placed in $2 \mathrm{ml}$ of buffer RLT (provided in the RNeasy kit), and sonicated for 45 seconds. Samples were then processed according to the manufacturer's protocol. Twenty milligrams of total RNA from each mouse were then processed using the RiboQuant Multi-Probe RNase Protection Assay System (PharMingen, San Diego, California, USA), using the mCK-5 multi-probe template set according to the manufacturer's protocol.

Ex vivo limiting dilution reactivation analysis. The frequency of cells capable of reactivating from latency was determined by a limiting dilution ex vivo reactivation assay as described previously $(15,17,20,22,23)$. Mechanical disrupted cells contained less than 1\% live cells, and thus the presence of preformed virus could be discerned from virus reactivating from latency as described previously $(15,17,20,22,23)$.

Limiting dilution PCR for viral genome-bearing cells. To detect peritoneal exudate cells and splenocytes harboring $\gamma \mathrm{HV} 68$ genome, a limiting dilution nested PCR assay was performed as described previously $(15,17,20$, $22,23)$. There were no false positives in the data in this report, and all assays demonstrated approximately one copy sensitivity per PCR reaction for plasmid DNA.

Statistical analysis. All data were analyzed using GraphPad Prism program (GraphPad Software for Science Inc., San Diego, California, USA) as described previously $(15,20)$. Titer data, virulence data, and differential counts were compared using a paired $t$ test. The frequencies of reactivation and genome-positive cells were analyzed by paired $t$ test. Frequencies of reactivation and genome-positive cells were obtained from cell numbers at which $63 \%$ of wells scored positive for either viral cytopathic effect or for presence of viral genome based on Poisson distribution. The data were then subjected 
a

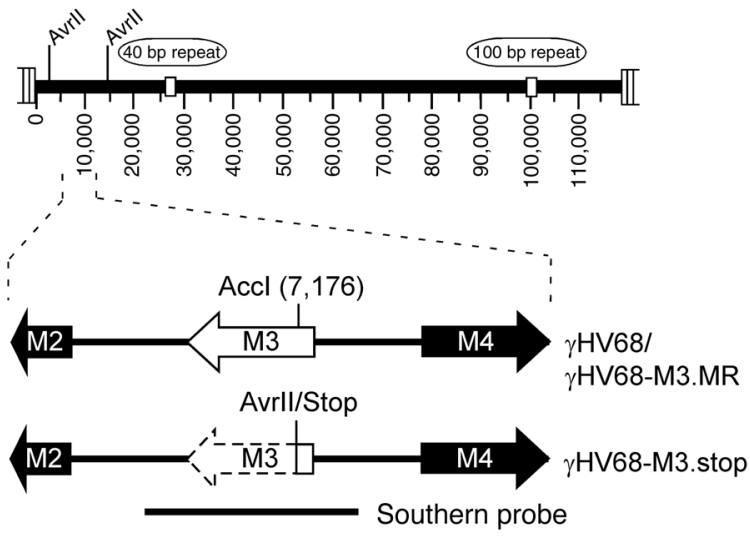

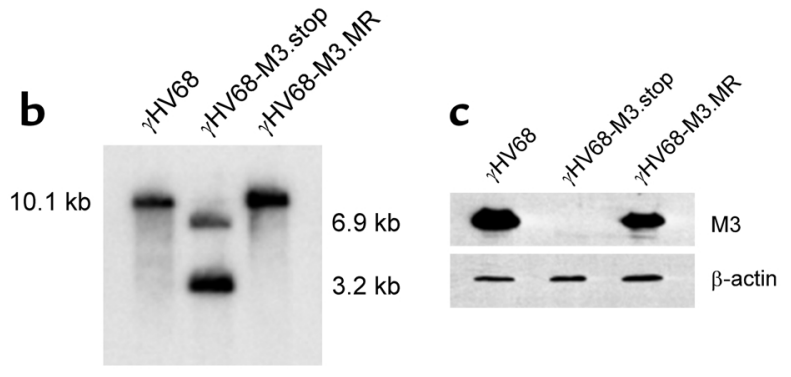

d

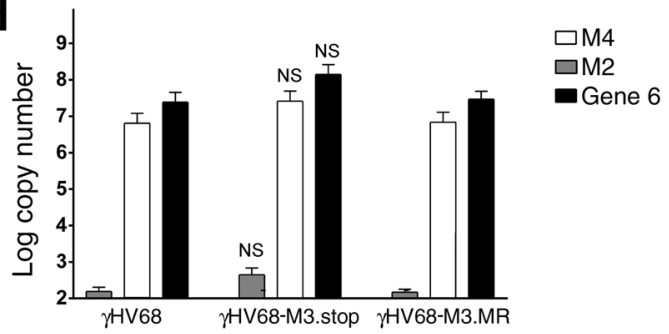

Figure 1

Construction and verification of the $\gamma H$ V68-M3.stop and $\gamma H V 68-M 3 . M R$ viruses. (a) Genomic structure of $\gamma H V 68, \gamma H V 68-M 3 . s t o p$, and $\gamma H V 68-M 3 . M R$ in the region of the M3 ORF. (b) Southern blot analysis of $\gamma H V 68, \gamma H V 68-M 3$.stop, and $\gamma H V 68-M 3$.MR. Viral DNA was purified from viral stocks, digested with Avrll, and analyzed with a probe spanning the M3 ORF (see a). The sizes of the hybridizing bands are indicated. (c) Western blot analysis of $\gamma \mathrm{HV} 68, \gamma \mathrm{HV} 68-\mathrm{M} 3$.stop, and $\gamma \mathrm{HV} 68-\mathrm{M} 3 . \mathrm{MR}$. Total cellular lysate was analyzed with either a polyclonal $\mathrm{Ab}$ to the $\mathrm{M} 3$ protein or an mAb to $\beta$-actin. (d) Real-time RT-PCR analysis of M2 and M4 genes in NIH 3 T12 cells lytically infected with either wild-type $\gamma H V 68, \gamma H V 68-M 3$.stop, or $\gamma H V 68-M 3 . M R$. Gene 6 transcription was analyzed in parallel as a control. Shown are mean \pm SEM of pooled data from at least three independent experiments. NS, no statistically significant difference between transcript levels in cells infected with $\gamma \mathrm{HV} 68-\mathrm{M} 3$. stop compared with cells infected with either $\gamma \mathrm{HV} 68$ or $\gamma \mathrm{HV} 68-\mathrm{M} 3 . \mathrm{MR}$.

to nonlinear regression analysis to obtain the single cell frequency for each limiting dilution analysis.

\section{Results}

Targeted disruption of the M3 open reading frame. We disrupted the M3 open reading frame (ORF) by inserting a translational stop with an accompanying frameshift mutation into the AccI site at genomic coordinate 7176 (18) ( $\gamma$ HV68-M3.stop virus; Figure 1a). This site is 34 amino acids into the 406 amino acids of the ORF, but is downstream from the end of the secretion signal peptide, such that truncated versions of the protein potentially made by downstream translation initiation should not be secreted from the cell.
Purification of $\gamma \mathrm{HV} 68$-M3.stop provided a homogenous stock, as determined by the presence of the inserted stop sequence in 50 out of 50 plaques (as determined by PCR analysis), absence of detectable M3 protein in 50 out of 50 plaques (as determined by ELISA), and appropriate digestion patterns on Southern analysis of 20 out of 20 plaques (data not shown). Southern analysis of $\gamma$ HV68-M3.stop by restriction digest with AvrII, using a ${ }^{32} \mathrm{P}-$ labeled M3 region probe (bp 5362-7893), demonstrated, as predicted, the addition of an additional AvrII site within the M3 ORF (Figure 1b). Western analysis of NIH 3T12 cells infected with the $\gamma$ HV68-M3.stop virus indicated that no full-length M3 protein, or truncated products (data

\section{Figure 2}

Replication of the $\gamma \mathrm{HV} 68-\mathrm{M} 3$.stop virus. (a) Multistep growth curve. NIH 3T12 monolayers were infected with $0.05 \mathrm{PFU}$ per cell of either $\gamma \mathrm{HV} 68$ or $\gamma \mathrm{HV} 68-\mathrm{M} 3$.stop, and samples were harvested at various times after infection. The mean and SEM of two independent experiments are shown. (b) C57BL/6 mice were infected with either $10^{6}$ PFU intraperitoneally or $4 \times 10^{5} \mathrm{PFU}$ intranasally and harvested at either 4 or 9 days after infection. Lung and spleen were assayed for viral titer. The mean \pm SEM of two independent experiments (ten mice total per condition) are shown. i.p., intraperitoneal; i.n., intranasal.
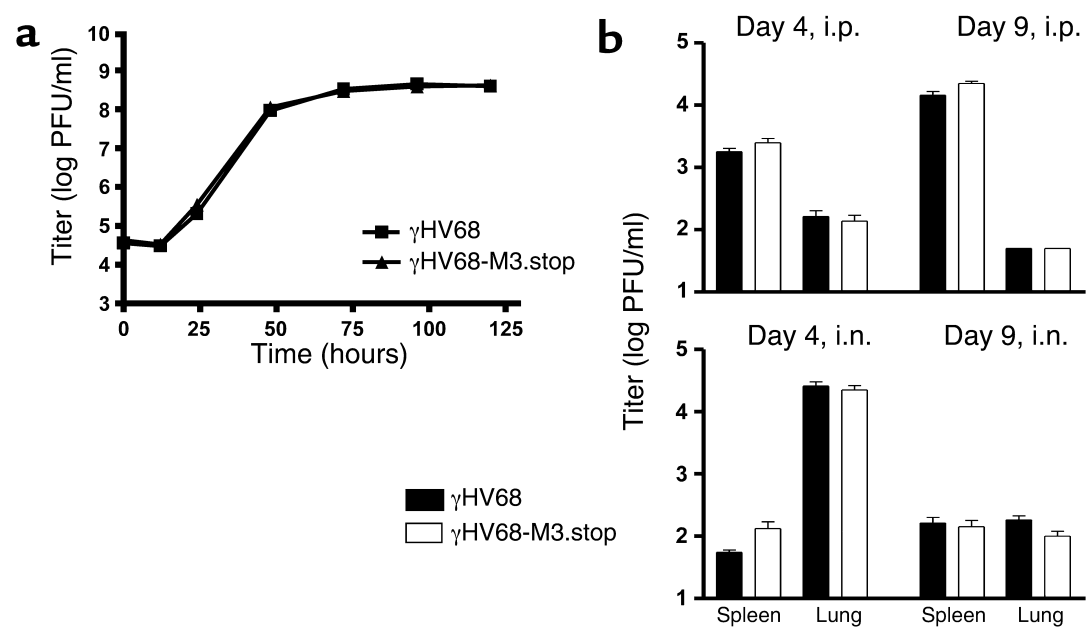


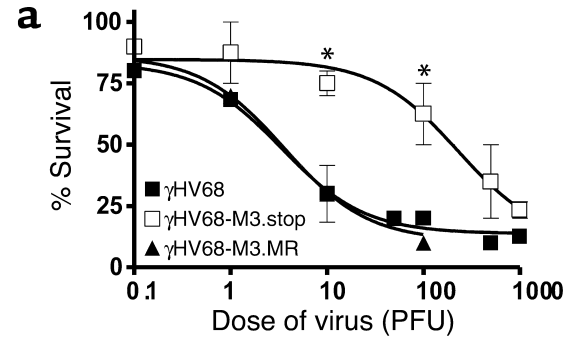

b

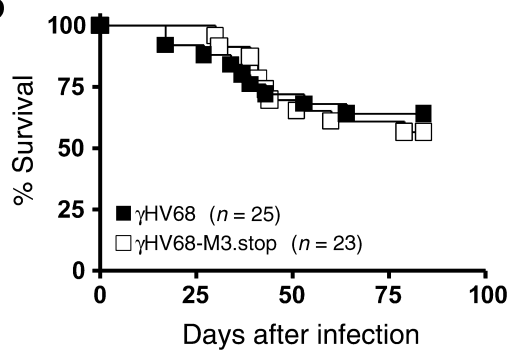

Figure 3

Virulence of $\gamma \mathrm{HV} 68-\mathrm{M} 3$.stop virus. (a) CD1 mice (21 days old) were inoculated intracerebrally with various doses of $\gamma \mathrm{HV} 68, \gamma \mathrm{HV} 68$ M3.stop, or $\gamma H$ V68-M3.MR, and followed for 2 weeks. Each data point represents the mean \pm SEM of three independent experiments (30 mice total per dose, $\left.{ }^{*} P<0.0001\right)$. (b) $\gamma \mathrm{IFNR}^{-/-}$mice $(n=$ number of mice across five separate experiments) were infected with $10^{6}$ PFU of either $\gamma \mathrm{HV} 68$ or $\gamma \mathrm{HV} 68-\mathrm{M} 3$.stop and followed for 84 days.

not shown), could be detected with a polyclonal $\mathrm{Ab}$ to the M3 protein (Figure 1c).

The M3 stop mutation lies $1,233 \mathrm{bp}$ from the predicted translational start of the adjacent $M 4$ gene and $2,548 \mathrm{bp}$ from the known transcriptional start site of the adjacent $M 2$ gene, making it unlikely that insertion of the M3 mutation would alter transcription of either M2 or M4 $(24,25)$. To determine if the M3.stop mutation altered $M 2$ or $M 4$ transcription, we performed realtime RT-PCR analysis of transcript levels in NIH 3 T12 fibroblasts infected with wild-type $\gamma \mathrm{HV} 688, \gamma \mathrm{HV} 68$ M3.stop, or $\gamma$ HV68-M3.MR. Transcript levels from the $M 2$ and $M 4$ genes were not significantly altered by the presence of the M3.stop mutation (Figure 1d).

To rule out the presence of distal mutations in $\gamma \mathrm{HV} 68$ M3.stop that might result in phenotypic alterations in $\gamma$ HV68-M3.stop, we constructed and purified a marker rescue virus ( $\gamma \mathrm{HV} 68-\mathrm{M} 3 . \mathrm{MR}$ ), which reconstituted wildtype M3 ORF sequence into $\gamma H V 68-M 3$.stop. Southern analysis of $\gamma$ HV68-M3.MR after AvrII digestion demonstrated, as expected, the loss of the engineered AvrII site (Figure 1b), and Western analysis indicated expression of the M3 protein (Figure 1c).

M3 is not required for efficient replication in cultured cells, spleen, or lung. Based on our ability to isolate the $\gamma \mathrm{HV} 68-$ M3.stop mutant, the M3 ORF is nonessential for in vitro replication. To evaluate whether $\mathrm{M} 3$ has a role in in vitro replication, we compared $\gamma \mathrm{HV} 68-\mathrm{M} 3$.stop and wild-type $\gamma \mathrm{HV} 68$ during multiple rounds of replication in NIH 3 T12 cells (Figure 2a). We found that M3 is not required for efficient replication in immortalized murine fibroblasts. Similarly, M3 was not required for efficient replication of $\gamma \mathrm{HV} 68$ in either the spleen or lung of C57BL/6 (B6) mice 4 or 9 days after either intraperitoneal or intranasal inoculation (Figure $2 b$ ).

$M 3$ is essential for efficient induction of meningitis and virulence after intracerebral inoculation. Chemokine expression has been demonstrated to increase after CNS infection with numerous viruses (26-29), and chemokines have been postulated to play an important role in CNS viral pathogenesis (reviewed in ref. 30). Considering that $\gamma \mathrm{HV} 68$ is capable of infecting numerous cell types within the brain (31), we tested the hypothesis that the M3 protein has a role after intracerebral infection of 21day-old immunocompetent CD1 mice. $\gamma H$ V68-M3.stop was 100 -fold less virulent than wild-type $\gamma \mathrm{HV} 68$ after intracerebral inoculation (Figure 3a). This attenuation was specific to the mutation in the M3 ORF, since $\gamma \mathrm{HV} 68-\mathrm{M} 3$.MR virus was equal in virulence to $\gamma \mathrm{HV} 68$. A decrease in virulence of the $\gamma \mathrm{HV} 68-\mathrm{M} 3$.stop compared with $\gamma \mathrm{HV} 68$-M3.MR after intracerebral inoculation was also demonstrated in $\mathrm{B} 6$ mice, assuring that the attenuated phenotype observed in CD1 mice is not strain dependent $(P=0.0263$, sixty 21 -day-old BL6 mice challenged with 1,10 , and $100 \mathrm{PFU}$ of $\gamma \mathrm{HV} 68$-M3.stop or $\gamma$ HV68-M3.MR).

We considered that M3 might be required for efficient replication in the brain despite the lack of a requirement for M3 in replication in the spleen and lung (above). We therefore determined the viral titer

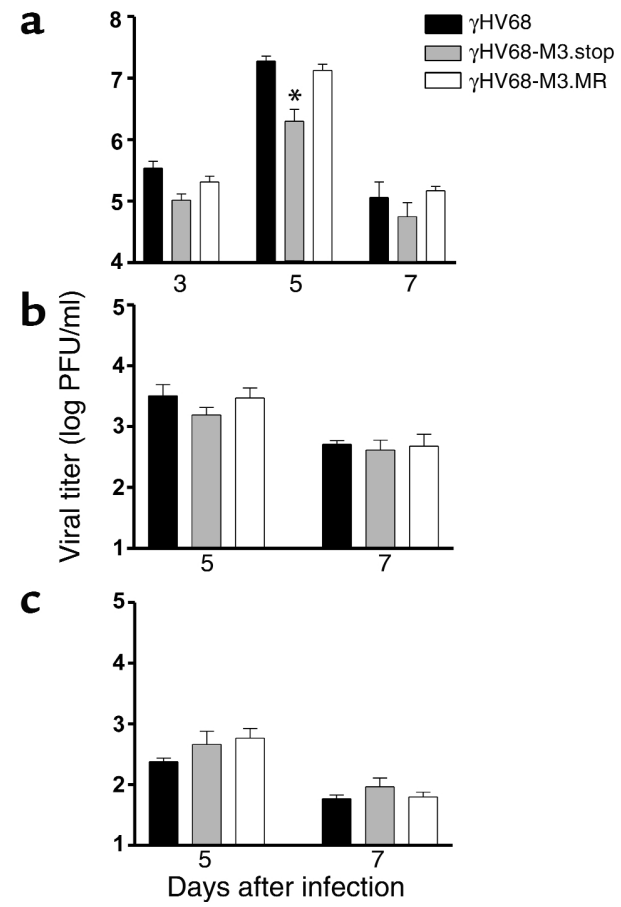

Figure 4

Viral titer after intracerebral infection with $\gamma \mathrm{HV} 68-\mathrm{M} 3$. stop virus. CD1 mice (21 days old) were inoculated intracerebrally with $100 \mathrm{PFU}$ of either $\gamma \mathrm{HV} 68, \gamma \mathrm{HV} 68-\mathrm{M} 3$.stop, or $\gamma \mathrm{HV} 68-\mathrm{M} 3 . M R$. At various points after infection, viral titer in the brain (a), spleen (b), or lung (c) was determined. Each data point represents the mean \pm SEM of two independent experiments (ten mice total per condition). ${ }^{*} P=0.0019$. 

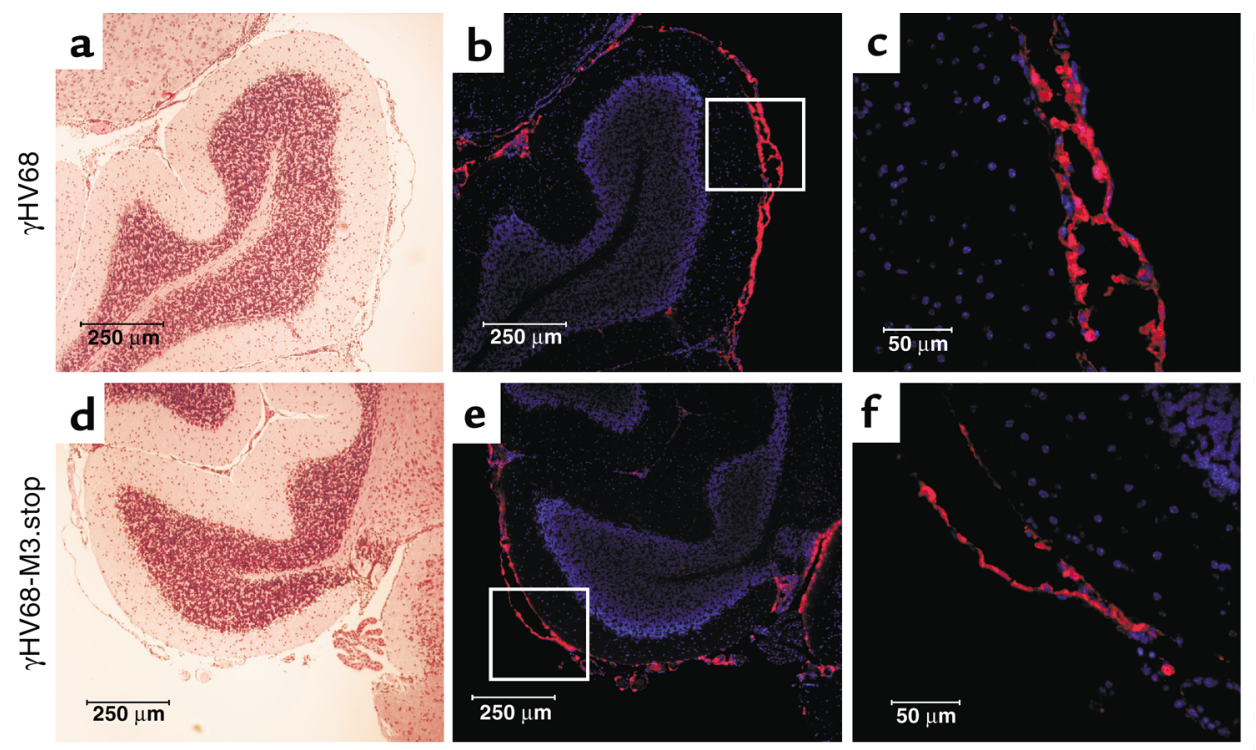

\begin{abstract}
Figure 5
Meningeal localization of viral antigen after infection with $\gamma \mathrm{HV} 68$ and $\gamma \mathrm{HV} 68-\mathrm{M} 3$.stop. Immunohistochemical localization of viral antigen after intracerebral inoculation of 21-day-old CD1 mice with 100 PFU of either $\gamma \mathrm{HV} 68$ (a-c) or $\gamma \mathrm{HV} 68$-M3.stop (d-f) demonstrates that viral antigen is present in the meninges of mice infected with either virus. (a and $\mathbf{d}$ ) Hematoxylin and eosin-stained sections of brain. (b and $\mathbf{e})$ Sections at same magnification, stained with a polyclonal Ab to $\gamma \mathrm{HV} 68$ and a Cy-3-conjugated secondary Ab (red). Slides were counterstained with Heochst dye, staining nuclei blue. (c and $\mathbf{f}$ ) Higher magnification of boxed areas in $\mathbf{b}$ and $\mathbf{e}$. Immunohistochemistry with preimmune serum as the primary $A b$ showed no reactivity with adjacent sections. Sections shown are representative examples of six mice, infected on two separate occasions. The scale bars represent $250 \mu \mathrm{m}$ for $\mathbf{a}, \mathbf{b}, \mathbf{d}$, and $\mathbf{e}$, and $50 \mu \mathrm{m}$ for $\mathbf{c}$ and $\mathbf{f}$.
\end{abstract}

within the brain, lung, and spleen at various days after intracerebral inoculation with $100 \mathrm{PFU}$ of either $\gamma$ HV68-M3.stop, $\gamma$ HV68, or $\gamma H V 68-M 3 . M R$. At the peak of viral replication in the brain (day 5; Figure 4a), there was approximately a tenfold decrease in viral titer in the brain that was specific to the mutation in the M3 ORF $(P=0.0019)$. This decrease was considerably smaller at day 7 after inoculation. Consistent with data after intraperitoneal or intranasal inoculation (Figure 2), after intracerebral inoculation there was no role for M3 in viral replication in lung or spleen (Figure 4, b and c).

To assess the location and extent of viral replication within the brain, as well as the nature of the inflammatory response to the infection, we performed histological examination of the brains of infected mice. Examination of sections at days 5 and 7 after infection indicated discontinuous areas of meningeal inflammation in mice infected with either $\gamma \mathrm{HV} 68$ or $\gamma \mathrm{HV} 68$ M3.stop. Meningeal inflammation was most prominent in the region of the cerebellum and basal forebrain. Examination of these slides with Ab specific to $\gamma \mathrm{HV} 68$ antigen indicated that virus was present at the sites of inflammation in similar patterns for both $\gamma \mathrm{HV} 68$ (Figure 5, a-c) and $\gamma$ HV68-M3.stop (Figure 5, d-f).

Strikingly, the nature of the inflammatory infiltrate was different after infection with $\gamma \mathrm{HV} 68$ compared with $\gamma$ HV68-M3.stop. $\gamma \mathrm{HV} 68$ and $\gamma$ HV68-M3.MR infection was associated with a marked preponderance of neutrophils in the meninges (Figure 6, a, c, d, and f). In contrast, the proportion of lymphocytes and macrophages was increased in the meninges of mice infected with $\gamma$ HV68-M3.stop (Figure 6, b and e). Differential counts conducted in a blinded manner confirmed that lack of M3 was associated with a considerable change in the inflammatory infiltrate (Figure 6g). Mutation of M3 led to a statistically significant increase in lymphocytes and macrophages ( $P=0.0449$ and $P=0.0004$, respectively) and a decrease in neutrophils $(P<0.0001)$ relative to infection with $\gamma$ HV68-M3.MR.

Chemokine expression is induced during $\gamma H V 68$-induced meningitis. Since M3 has an important role in neurovirulence and is a high-affinity CBP, we wished to determine whether M3 ligands are expressed during CNS infection with $\gamma \mathrm{HV} 68$. We therefore performed a RNase protection assay to determine whether chemokine expression increases in response to infection with $\gamma \mathrm{HV} 68$. As shown in Figure 7, there was no detectable expression of any of the chemokines assayed in either naive mice or in mice 5 days after mock infection. In contrast, expression of CC chemokines RANTES, MIP- $1 \beta$, and MCP- 1 , as well as the CXC chemokine IP-10, were detected 5 days after infection with either $\gamma$ HV68-M3.MR or $\gamma H$ HV68M3.stop (Figure 7). These data support the hypothesis that M3 may regulate CNS disease via its capacity to sequester inflammatory chemokines.

M3 does not play a role in regulating chronic inflammation in immunocompromised mice. Since M3 is important for regulating inflammatory responses during acute $\gamma \mathrm{HV} 68$ infection, we considered the possibility that the M3 protein may regulate chronic inflammatory responses to $\gamma \mathrm{HV} 68$ infection. We therefore examined 
the role of M3 in inflammatory aortitis and splenic atrophy induced by $\gamma \mathrm{HV} 68$ in IFN $\gamma \mathrm{R}^{-/-}$mice infected for weeks to months with either $\gamma \mathrm{HV} 68$-M3.stop or $\gamma \mathrm{HV} 68(21,32)$. After infection with $2 \times 10^{6} \mathrm{PFU}$ of either $\gamma \mathrm{HV} 68-\mathrm{M} 3$.stop or $\gamma \mathrm{HV} 68, \mathrm{INF}^{-/-}$mice died with similar kinetics and frequency (Figure $3 b$ ). Histological examination of aortic inflammation and splenic atrophy in these mice indicated that M3 plays no role in either the penetrance or severity of splenic or aortic lesions caused by either virus (data not shown).

$M 3$ is not required for establishment or reactivation from latency. Since the region of the M3 ORF is transcribed in latently infected tissues (33-35) and a recent study indicated a $\gamma \mathrm{HV} 68$ mutant virus in which a LacZ cassette was placed in the $M 3$ gene has a marked reduction in establishment of latency (36), we examined whether $\gamma$ HV68-M3.stop was capable of establishing a latent infection in $\mathrm{B} 6$ mice after either intranasal or intraperitoneal inoculation. At either 2 weeks (Figure 8 , a-d) or 7 weeks after infection (data not shown), splenocytes and peritoneal exudate cells were assessed for their ability to reactivate from latency in an ex vivo limiting dilution analysis $(22,23)$. We found that similar frequencies of reactivating cells were recovered after infection with either $\gamma \mathrm{HV} 68$-M3.stop or $\gamma \mathrm{HV} 68$, regardless of route of inoculation. To measure persistent replication in samples, replicate cell aliquots were mechanically disrupted. This procedure kills more than $99 \%$ of cells, but has at most a twofold effect on viral titer (22), thus allowing experimental distinction between reactivation from latency (which requires live cells) and persistent replication of virus in tissues. No significant persistent replication of either virus was detected under any of the experimental conditions (Figure 8, a-d).

Using a nested PCR to detect $\gamma \mathrm{HV} 68$ genome-bearing (15) cells, we found that at 2 weeks after infection, similar frequencies of cells harbored $\gamma \mathrm{HV} 68$ genome in both splenocytes and peritoneal exudate cells after infection with either $\gamma$ HV68-M3.stop or $\gamma \mathrm{HV} 68$ (Figure 8, e-h), demonstrating that the $\mathrm{M} 3$ protein is not required for establishment of or reactivation from latency.
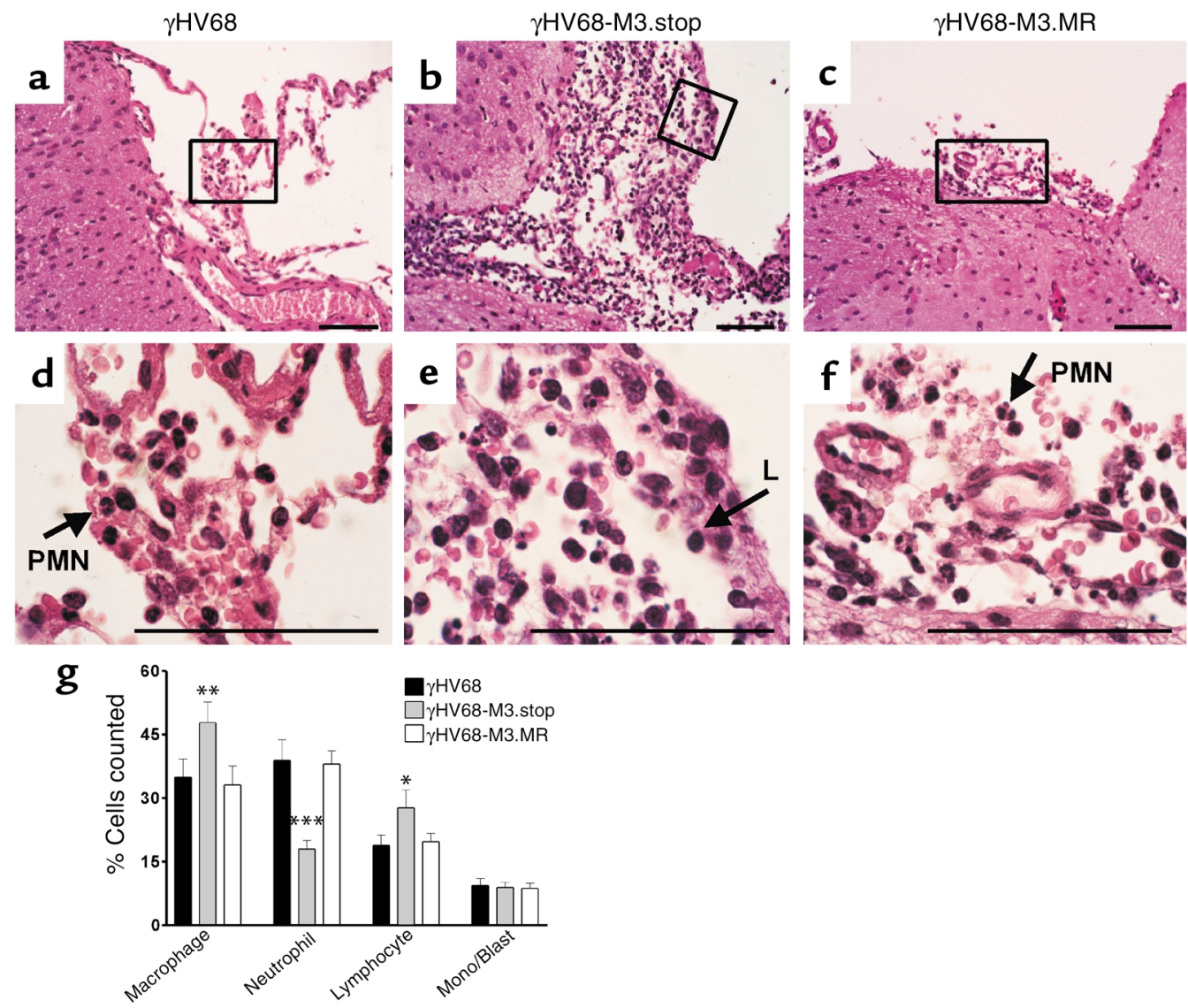

Figure 6

The M3 protein alters the inflammatory response to $\gamma H V 68$. Hematoxylin and eosin-stained sagital brain sections from the basal forebrain of CD1 mice infected with $\gamma \mathrm{HV} 68$ ( $\mathbf{a}$ and $\mathbf{d}$ ), $\gamma \mathrm{HV} 68-\mathrm{M} 3$.stop ( $\mathbf{b}$ and $\mathbf{e}$ ), or $\gamma \mathrm{HV} 68-\mathrm{M} 3 . \mathrm{MR}$ ( $\mathbf{c}$ and $\mathbf{f}) 5$ days previously. The boxed areas in $\mathbf{a}-\mathbf{c}$ are shown at higher magnification in $\mathbf{d}-\mathbf{f}$. The arrows in $\mathbf{d}$ and $\mathbf{f}$ indicate neutrophils (PMN). The arrow in $\mathbf{e}$ indicates a lymphocyte (L). (g) Differential counts of meningeal infiltrates of mice infected 5 days previously with the indicated viruses. Shown are mean \pm SEM of pooled numbers from two independent investigators performing differential counts in a blinded manner on sections from four mice infected with $\gamma \mathrm{HV} 68$, eight mice infected with $\gamma \mathrm{HV} 68-\mathrm{M} 3$.MR, and eight mice infected with $\gamma \mathrm{HV} 68-\mathrm{M} 3 . \operatorname{stop}\left({ }^{*} P=0.0449,{ }^{*} P=0.0004\right.$, and $\left.{ }^{* * *} P<0.0001\right)$. Scale bars, $100 \mu \mathrm{m}$. Mono/Blast, monocytes or lymphoblasts. 


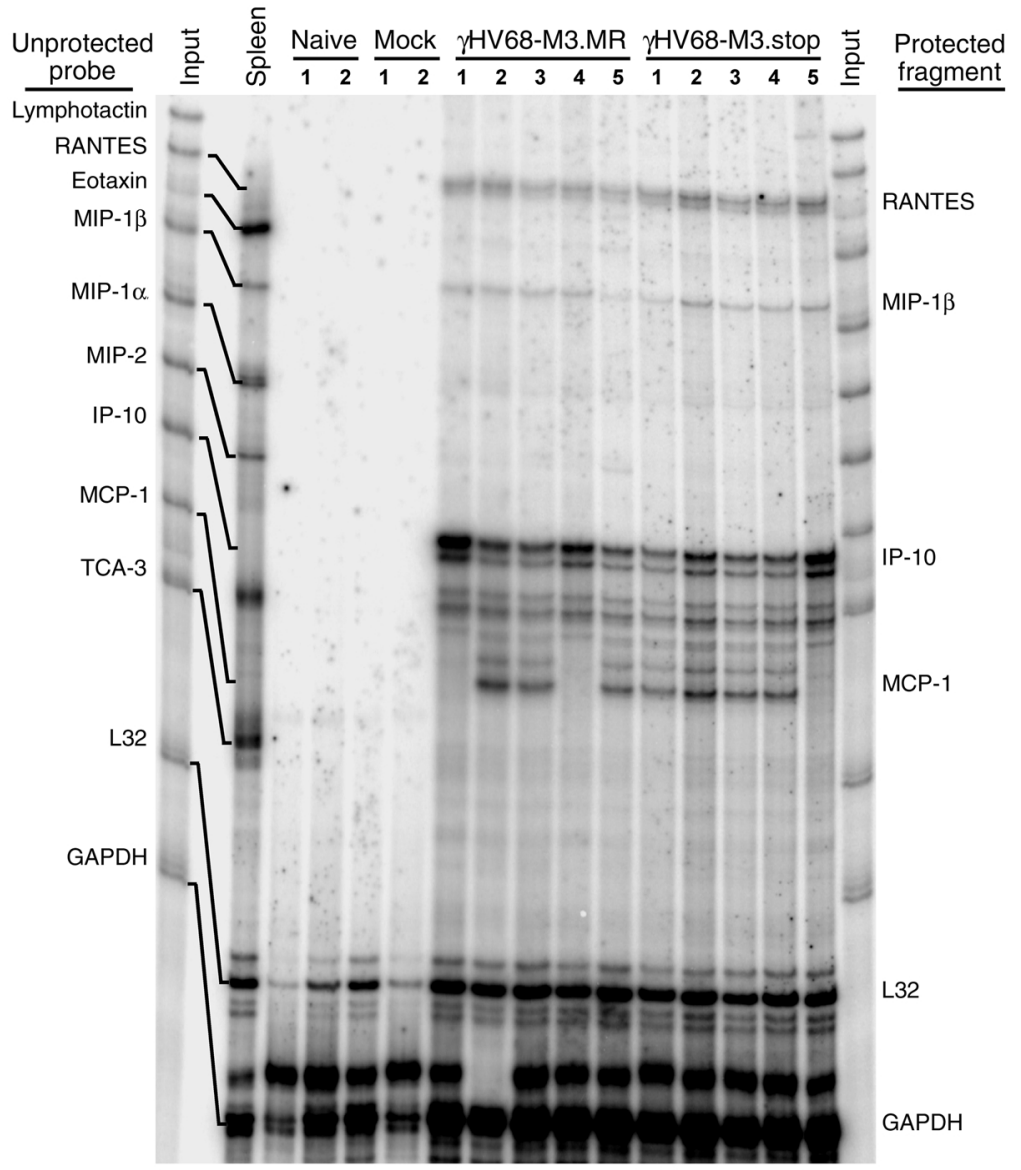

\section{Figure 7}

Enhanced chemokine mRNA expression after intracerebral inoculation with $\gamma \mathrm{HV} 68$. RNA was harvested from brains of naive mice, mock-infected mice, or mice infected with 100 PFU of either $\gamma \mathrm{HV} 68-\mathrm{M} 3 . \mathrm{MR}$ or $\gamma \mathrm{HV} 68-\mathrm{M} 3$.stop and subjected to RNase protection analysis. Twenty micrograms of total RNA were added per sample, except for the positive control mouse splenocyte RNA lane, which contained $2 \mu \mathrm{g}$ RNA. Differences in loading between lanes were at most tenfold (e.g., compare L32 in Mock, lane 2, with $\gamma \mathrm{HV} 68-\mathrm{M} 3 . \mathrm{MR}$, lane 1), while differences in expression of chemokines were in the range of 600-fold (e.g., compare RANTES in Mock, lane 2, with $\gamma$ HV68-M3.MR, lane 1). Band intensity was quantified using the STORM Phosphoimager software package (Molecular Dynamics, Piscataway, New Jersey, USA).

\section{Discussion}

The coordination of leukocyte recruitment into sites of infection is a critical aspect of the inflammatory response. Chemokines play a central role in this process through the activation and mobilization of macrophages, lymphocytes, dendritic cells, natural killer cells, and granulocytes (37). Recently, a number of reports have documented the possible involvement of chemokines in the immunopathogenesis of different infectious diseases of the CNS (26-29). Our data is most consistent with a role for $\mathrm{CC}$ chemokines in protecting the CNS from viral infection. Moreover, secretion of a selective high-affinity CBP by a herpesvirus suggests that soluble chemokines play a central role in herpesvirus infection. We have examined, through the use of a viral mutant lacking the M3 protein, the role of chemokines in acute, chronic, and latent infection with the $\gamma 2$-herpesvirus $\gamma \mathrm{HV} 68$.
Absence of the M3 protein from $\gamma \mathrm{HV} 68$ infection resulted in a significantly attenuated phenotype, but notably only under specific circumstances. There was no apparent defect in the ability of the virus to replicate or spread from the site of infection to distal organs after either intraperitoneal or intranasal inoculation, indicating that either chemokines do not contribute to viral clearance under these conditions or that the virus contains other proteins that can compensate for the lack of the M3 protein. In contrast to this result, the M3-deficient virus demonstrated decreased virulence after intracerebral inoculation, with a concomitant decrease in viral titer. These data, combined with the fact that both wild-type and M3-deficient viruses replicated to similar levels in distal organs after intracerebral inoculation, suggest that chemokines may have a greater role in protection of the CNS from herpesvirus infection than other organ systems within the host.

The predominately neutrophilic infiltrate associated with infection in the presence of the M3 protein, compared with the relative abundance of lymphocytes and macrophages after infection with a virus lacking the M3 protein, shows that the M3 protein significantly alters the inflammatory response to $\gamma \mathrm{HV} 68$. Whether these alterations in inflammation are dependent on, or independent of, the effects of M3 on viral replication is not known. However, examination of the chemokines typically produced during CNS infection suggests a mechanism by which M3 could alter the inflammatory response and thereby contribute to virulence. In experimental and clinical bacterial meningoencephalitis there is dominant cerebral production of the neutrophil and monocyte attractant chemokines IL-8, MIP-2, GRO $\alpha$, MCP-1, MIP- $1 \alpha$, and MIP- $1 \beta$, and the inflammatory infiltrates consist of predominantly neutrophils and monocytes (38-40). In contrast, during viral meningoencephalitis, including $\gamma \mathrm{HV} 68$ (Figure 5 and 6), cerebral expression of the lymphocyte and monocyte chemoattractants IP-10, MCP-1, and RANTES predominates (29). This pattern of chemokine expression would be predicted to induce an infiltrate consisting primarily of lymphocytes and monocytes (30). The M3 protein is capable of binding to MIP- $1 \alpha$, MCP- 1 , and RANTES, while it does not bind with appreciable affinity to IL-8, MIP-2, GRO $\alpha$, or IP-10 (8). Thus, by binding to and antagonizing the activity of chemokines such as 
MIP-1 $\alpha$, MCP-1, and RANTES, the M3 protein could shift the inflammatory response from lymphocytes and monocytes to neutrophils, which may be less effective at controlling viral infection.

Data from these studies demonstrate no role for the M3 protein in two aspects of chronic infection-induction of aortic inflammation in IFN- $\gamma$-unresponsive mice and in the establishment, maintenance of, or reactivation from latency. Consistent with the idea that the M3 protein has a role only in limited aspects of $\gamma \mathrm{HV} 68$ infection, two measures of chronic disease - vasculitis and splenic atrophy in IFN- $\gamma$-unresponsive mice were unchanged after infection with a virus deficient in production of the M3 protein (data not shown). It is intriguing that the data presented here demonstrate no role for the M3 protein in either establishment, maintenance, or reactivation from latency, despite a recent study indicating that a virus containing a LacZ insertion disrupting the M3 ORF ( $\gamma$ HV68-M3LacZ) has a dramatic reduction in establishment of latency (36). It is possible that the phenotype of $\gamma$ HV68-M3LacZ reported by Bridgeman et al. (36) is due to the insertion of a large transcriptionally active cassette in the $M 3$ region rather than deletion of M3. This is consistent with findings showing that insertion of a Lac $Z$ cassette into the $\gamma \mathrm{HV} 68 \mathrm{M} 1$ locus generated viral phenotypes not attributable to deletion of M1 (15). It is possible that the presence of the LacZ cassette in the $M 3$ locus alters the expression of other $\gamma \mathrm{HV} 68$ genes, and it is these other gene products that are responsible for the altered phenotype. For example, the phenotype of a stop mutant in the $M 2$ gene (19), which is adjacent to the $M 3$ gene, is very similar to the phenotype reported by Bridgeman et al. for $\gamma \mathrm{HV} 68$-M3LacZ, raising the possibility that the phenotype attributed to M3 by Bridgeman et al. is due to alterations in M2. Insertion of the M3.stop mutation did not alter transcript levels from either $M 2$ or $M 4$ (Figure 1d).

The lack of a role for M3 in latency is somewhat surprising since several studies have indicated that the region of the $M 3 \mathrm{ORF}$ is transcriptionally active during latency (33-35). There are several possible interpretations of these data. First, we have found previously that the timing of transcription is not predictive of the function of a gene in vivo. For example, the v-cyclin is an abundantly transcribed lytic cycle gene, but its primary role in vivo is in the regulation of reactivation from latency $(20,41)$. Second, transcriptional assays may be indicating that the locus surrounding the M3 ORF is active during latency, with a gene product other than the M3 protein critical for establishment of latency. Third, it is possible that the M3 protein has a role in latency that has not been demonstrated by the assays described here. Finally, it is possible that the latent populations examined for transcriptional activity contain a few cells that are replicating virus. The relative abundance of the $M 3$ transcript (16) might lead to it scoring positive in a transcriptional assay while other lytic transcripts remain below the limit of detection. It is important to note that despite the lack of a role for M3 in latency and reactivation, it is possible that the M3 protein does have a role in chronic infection that is as yet undetected.

This work shows that the M3 protein has a potent capacity to regulate inflammation in vivo, resulting in increased virulence during acute infection. The capacity to inhibit lymphocyte-dependent inflammation raises the possibility that M3 may alter inflammatory processes that are induced by CC chemokines without compromising all chemokine-based host responses. Since M3 can inhibit CC chemokine-dependent responses, M3 may be useful clinically to combat diseases in which lymphocytes and macrophages cause tissue destruction. M3 may also provide a tool for analyzing the role of chemokines in other experimental models of disease.

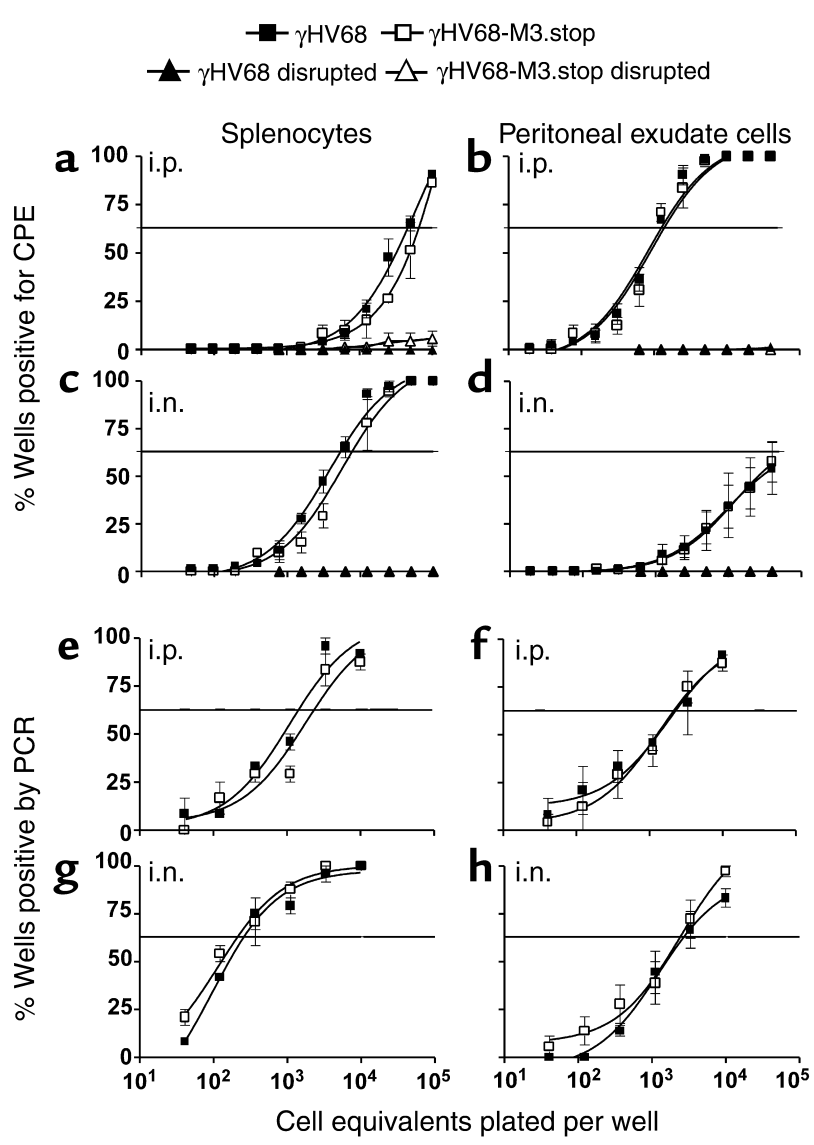

Figure 8

$\gamma \mathrm{HV} 68-\mathrm{M} 3$.stop establishes and reactivates from latency normally. Sixteen days after inoculation with either $10^{6} \mathrm{PFU}$ intraperitoneally (a, b, e, and $\mathbf{f}$ ) or $4 \times 10^{5}$ PFU intranasally (c, d, $\mathbf{g}$, and $\mathbf{h}$ ), splenocytes (a, c, e, and $\mathbf{g}$ ) and peritoneal exudate cells $(\mathbf{b}, \mathbf{d}, \mathbf{f}$, and $\mathbf{h})$ were harvested from B6 mice. Ex vivo reactivation ( $\mathbf{a}, \mathbf{b}, \mathbf{c}$, and $\mathbf{d}$ ) was monitored by plating cells over an indicator monolayer and monitoring for viral cytopathic effect 21 days after plating (square symbols). Duplicate cell samples were mechanically disrupted to control for persistent viral replication (triangle symbols, denoted as disrupted in the figure). (e, $\mathbf{f}, \mathbf{g}$, and $\mathbf{h}$ ) Percentage of cells harboring viral genome was assessed through a limiting dilution nested PCR assay. Data are pooled from three independent experiments of five mice per infectious condition (15 mice total per condition). 


\section{Acknowledgments}

This work was supported by NIH grants CA-74730 and HL-60090 to H.W. Virgin IV. S.H. Speck was supported by NIH grants CA-43143, CA-52004, and CA-58524. V. van Berkel was supported by NIH grant GM-07200. B. Levine was supported by NIH grant A1-44157. S.B. Kapadia was supported by a predoctoral training grant in tumor immunology from the Cancer Research Institute. We thank Daved Fremont, David Leib, the members of his laboratory, and the members of the Speck and Virgin laboratories for their helpful comments during the course of this research.

1. Schall, T.J. and Bacon, K.B. 1994. Chemokines, leukocyte trafficking, and inflammation. Curr. Opin. Immunol. 6:865-873.

2. Penfold, M.E., et al. 1999. Cytomegalovirus encodes a potent alpha chemokine. Proc. Natl. Acad. Sci. USA. 96:9839-9844.

3. Gao, J.L., and Murphy, P.M. 1994. Human cytomegalovirus open reading frame US28 encodes a functional $\beta$ chemokine receptor. J. Biol. Chem. 269:28539-28542.

4. MacDonald, M.R., Li, X.-Y., and Virgin, H.W. 1997. Late expression of a $\beta$ chemokine homolog by murine cytomegalovirus. J. Virol. 71:1671-1678.

5. Lalani, A.S., Barrett, J., and McFadden, G. 2000. Modulating chemokines: more lessons from viruses. Immunol. Today. 21:526-527.

6. Lalani, A.S., and McFadden, G. 1997. Secreted poxvirus chemokine binding proteins. J. Leukoc. Biol. 62:570-576.

7. Parry, B.C., et al. 2000. A broad spectrum secreted chemokine binding protein encoded by a herpesvirus. J. Exp. Med. 191:573-578.

8. van Berkel, V., et al. 2000. Identification of a gammaherpesvirus selective chemokine binding protein that inhibits chemokine action. J. Virol. 74:6741-6747

9. Mossman, K., et al. 1996. Myxoma virus M-T7, a secreted homolog of the interferon-gamma receptor, is a critical virulence factor for the development of myxomatosis in European rabbits. Virology. 215:17-30.

10. Lalani, A.S., et al. 1999. Role of the myxoma virus soluble CC-chemokine inhibitor glycoprotein, M- T1, during myxoma virus pathogenesis. Virology. 256:233-245.

11. Graham, K.A., et al. 1997. The T1/35kDa family of poxvirus-secreted proteins bind chemokines and modulate leukocyte influx into virus-infected tissues. Virology. 229:12-24.

12. Virgin, H.W., and Speck, S.H. 1999. Unraveling immunity to gamma-herpesviruses: a new model for understanding the role of immunity in chronic virus infection. Curr. Opin. Immunol. 11:371-379.

13. Speck, S.H., and Virgin, H.W. 1999. Host and viral genetics of chronic infection: a mouse model of gamma-herpesvirus pathogenesis. Curr. Opin. Microbiol. 2:403-409.

14. Simas, J.P., Bowden, R.J., Paige, V., and Efstathiou, S. 1998. Four tRNAlike sequences and a serpin homologue encoded by murine gammaherpesvirus 68 are dispensable for lytic replication in vitro and latency in vivo. J. Gen. Virol. 79:149-153.

15. Clambey, E.T., Virgin, H.W., and Speck, S.H. 2000. Disruption of the murine gammaherpesvirus $68 \mathrm{M} 1$ open reading frame leads to enhanced reactivation from latency. J. Virol. 74:1973-1984.

16. van Berkel, V., Preiter, K., Virgin, H.W., and Speck, S.H. 1999. Identification and initial characterization of a murine gammaherpesvirus 68 gene encoding an abundantly secreted protein. J. Virol. 73:4524-4529.

17. Weck, K.E., Barkon, M.L., Yoo, L.I., Speck, S.H., and Virgin, H.W. 1996 Mature B cells are required for acute splenic infection, but not for establishment of latency, by murine gammaherpesvirus 68. J. Virol. 70:6775-6780

18. Virgin, H.W., et al. 1997. Complete sequence and genomic analysis of murine gammaherpesvirus 68. J. Virol. 71:5894-5904.

19. Jacoby, M.A., Virgin, H.W., IV, and Speck, S.H. 2002. Disruption of the M2 gene of murine gammaherpesvirus 68 alters splenic latency follow- ing intranasal, but not intraperitoneal, inoculation. J. Virol. 76:1790-1801.

20. Van Dyk, L.F., Virgin, H.W., and Speck, S.H. 2000. The murine gammaherpesvirus $68 \mathrm{v}$-cyclin is a critical regulator of reactivation from latency. J. Virol. 74:7451-7461.

21. Weck, K.E., et al. 1997. Murine gammaherpesvirus 68 causes large vesse arteritis in mice lacking interferon-gamma responsiveness: a new model for virus induced vascular disease. Nat. Med. 3:1346-1353.

22. Weck, K.E., Kim, S.S., Virgin, H.W., and Speck, S.H. 1999. B cells regulate murine gammaherpesvirus 68 latency. J. Virol. 73:4651-4661.

23. Weck, K.E., Kim, S.S., Virgin, H.W., and Speck, S.H. 1999. Macrophages are the major reservoir of latent murine gammaherpesvirus 68 in peritoneal cells. J. Virol. 73:3273-3283.

24. Kapadia, S.B., Molina, H., van Berkel, V., Speck, S.H., and Virgin, H.W. 1999. Murine gammaherpesvirus 68 encodes a functional regulator of complement activation. J. Virol. 73:7658-7670.

25. Husain, S.M., et al. 1999. Murine gammaherpesvirus M2 gene is latency-associated and its protein a target for CD8(+) T lymphocytes. Proc. Natl. Acad. Sci. USA. 96:7508-7513.

26. Palma, J.P., and Kim, B.S. 2001. Induction of selected chemokines in glial cells infected with Theiler's virus. J. Neuroimmunol. 117:166-170.

27. Liu, M.T., Armstrong, D., Hamilton, T.A., and Lane, T.E. 2001. Expression of Mig (monokine induced by interferon-gamma) is important in $\mathrm{T}$ lymphocyte recruitment and host defense following viral infection of the central nervous system. J. Immunol. 166:1790-1795.

28. Asensio, V.C., and Campbell, I.L. 1997. Chemokine gene expression in the brains of mice with lymphocytic choriomeningitis. J. Virol. 71:7832-7840.

29. Lane, T.E., et al. 1998. Dynamic regulation of alpha- and beta-chemokine expression in the central nervous system during mouse hepatitis virusinduced demyelinating disease. J. Immunol. 160:970-978.

30. Campbell, I.L. 2000. Cytokines and chemokines in defense and damage in the intact CNS. In New concepts in the immunopathogenesis of CNS infections. P.K. Peterson and J.S. Remington, editors. Blackwell Science Inc. Malden, Massachusetts, USA. 51-83.

31. Terry, L.A., Stewart, J.P., Nash, A.A., and Fazakerley, J.K. 2000. Murine gammaherpesvirus-68 infection of and persistence in the central nervous system. J. Gen. Virol. 81:2635-2643.

32. Dutia, B.M., Clarke, C.J., Allen, D.J., and Nash, A.A. 1997. Pathological changes in the spleens of gamma interferon receptor-deficient mice infected with murine gammaherpesvirus: a role for CD8 T cells. J. Virol. 71:4278-4283.

33. Virgin, H.W., Presti, R.M., Li, X.-Y., Liu, C., and Speck, S.H. 1999. Three distinct regions of the murine gammaherpesvirus 68 genome are transcriptionally active in latently infected mice. J. Virol. 73:2321-2332.

34. Simas, J.P., Swann, D., Bowden, R., and Efstathiou, S. 1999. Analysis of murine gammaherpesvirus-68 transcription during lytic and latent infection. J. Gen. Virol. 80:75-82.

35. Rochford, R., Lutzke, M.L., Alfinito, R.S., Clavo, A., and Cardin, R.D. 2001. Kinetics of murine gammaherpesvirus 68 gene expression following infection of murine cells in culture and in mice. J. Virol. 75:4955-4963.

36. Bridgeman, A., Stevenson, P.G., Simas, J.P., and Efstathiou, S. 2001. A secreted chemokine binding protein encoded by murine gammaherpesvirus-68 is necessary for the establishment of a normal latent load. $J$ Exp. Med. 194:301-312.

37. Luster, A.D. 1998. Chemokines - chemotactic cytokines that mediate inflammation. N. Engl. J. Med. 338:436-445.

38. Sprenger, H., et al. 1996. Chemokines in the cerebrospinal fluid of patients with meningitis. Clin. Immunol. Immunopathol. 80:155-161.

39. Spanaus, K.S., et al. 1997. C-X-C and C-C chemokines are expressed in the cerebrospinal fluid in bacterial meningitis and mediate chemotactic activity on peripheral blood-derived polymorphonuclear and mononuclear cells in vitro. J. Immunol. 158:1956-1964.

40. Lahrtz, F., Piali, L., Spanaus, K.S., Seebach, J., and Fontana, A. 1998. Chemokines and chemotaxis of leukocytes in infectious meningitis. $J$. Neuroimmunol. 85:33-43.

41. Hoge, A.T., Hendrickson, S.B., and Burns, W.H. 2000. Murine gammaherpesvirus 68 cyclin D homologue is required for efficient reactivation from latency. J. Virol. 74:7016-7023. 